\title{
Conversion of the CD8 lineage to CD4 T cells
}

Editorial

\section{Maria P. Guzman and Zhibin Chen}

Mature $\mathrm{T}$ lymphocytes possess a CD4 or CD8 lineage-specific marker. These molecules also serve as a co-receptor which along with a specific T Cell Receptor (TCR) binds to MHC class II (MHCII) or class I (MHCI), respectively. MHCI is expressed by all nucleated cells, whereas MHCII by antigen-presenting cells (APC). CD8 $\mathrm{T}$ cells kill target cells through MHCI-based recognition. CD4 T cells recognize MHCII and act either as helper T (Th) cells potentiating immunity or as regulatory $\mathrm{T}$ (Treg) cells inducing tolerance.

The development of the adaptive immune repertoire is based on discrimination of self-antigens in the host against nonself-antigens from foreign invaders [1]. This concept has been serving as a pillar for immunology but faces a challenge to accommodate the interplay between the host immune system and the mutualistic microbiota. We tracked the clonal fate of CD4 and CD8 T cells at the interface with gut microbiota. We found that CD8 T cells cross-differentiated into MHCI-restricted CD4 $\mathrm{T}_{h}$ cells or Foxp3 ${ }^{+} \mathrm{T}_{\text {reg }}$ cells [2] (Figure 1). The CD8 lineage plasticity was found in two independent models of TCRtrangenic mice (OT1 and 8.3) and natural CD8 T cells from wildtype mice. The conversion from CD8 T cells to MHCI-restricted CD4 $\mathrm{T}_{\text {reg }}$ cells occurred in the gutassociated environment without regard to self-antigens, with a host-intrinsic plasticity amplified by microbiota. The MHCI- $\mathrm{T}_{\text {reg }}$ cell, in its physiological niche in the gut lamina propria or in a setting of adoptive transfer, potently suppressed inflammatory damage even in the apparent absence of cognate antigens [2].

Why is such an intrinsic plasticity built in the host immune system during evolution? The T cell repertoire consists of CD4 and CD8 T cells generated by thymic selection in newborns. With age, the thymus ceases to function but the thymic-derived $\mathrm{T}$ cell repertoire largely persists. In certain diseases, a portion or a subtype of $T$ cells might be lost. Conceivably, evolution may have endowed mechanisms to restore the immune balance to cope with catastrophic damages, such as T cell depletion by viral infections or natural irradiation. The plasticity of the CD8 T cell lineage elucidated by our study has perhaps evolved as an alternative pathway to protect the integrity of the adaptive immune system. In the adverse event of CD4 T cell depletion, CD4 populations can be replenished via cross-differentiation from CD8 T cells. One of the fascinating aspects of this transition is the requirement of MHCII despite that the clonotype TCR recognizes MHCIpresentated antigens [2].
How is the CD8-to-CD4 lineage plasticity relevant to biomedicine? In the modern world, HIV infection perhaps represents the most known example of catastrophic loss of $\mathrm{CD} 4 \mathrm{~T}$ cells and subsequent imbalance of the $\mathrm{CD} 8$ versus $\mathrm{CD} 4$ lineages. It remains to be seen whether the depletion of CD4 T cells in HIV infection triggers cross-differentiation from the CD8 lineage to CD4 $\mathrm{T}$ cells. If so, the conversion might not only replenish the CD4 T cell pool with new targets for infection by HIV but also exacerbate the depletion of CD8 populations, while creating a potent immunosuppression in the gut mucosa by the converted MHCI-T $\mathrm{T}_{\text {reg }}$ cells.

An imbalance of $\mathrm{CD} 8$ versus CD4 $\mathrm{T}$ cells could also be medically introduced in a type of cancer immunotherapy, wherein a patient is subject to immunoablation conditioning and then receives adoptive transfer of CD8 T cells enriched for specificities against tumors. Tumors present an immunoprivileged microenvironment with some neoantigens but mostly self-antigens [3]. It remains to be examined whether

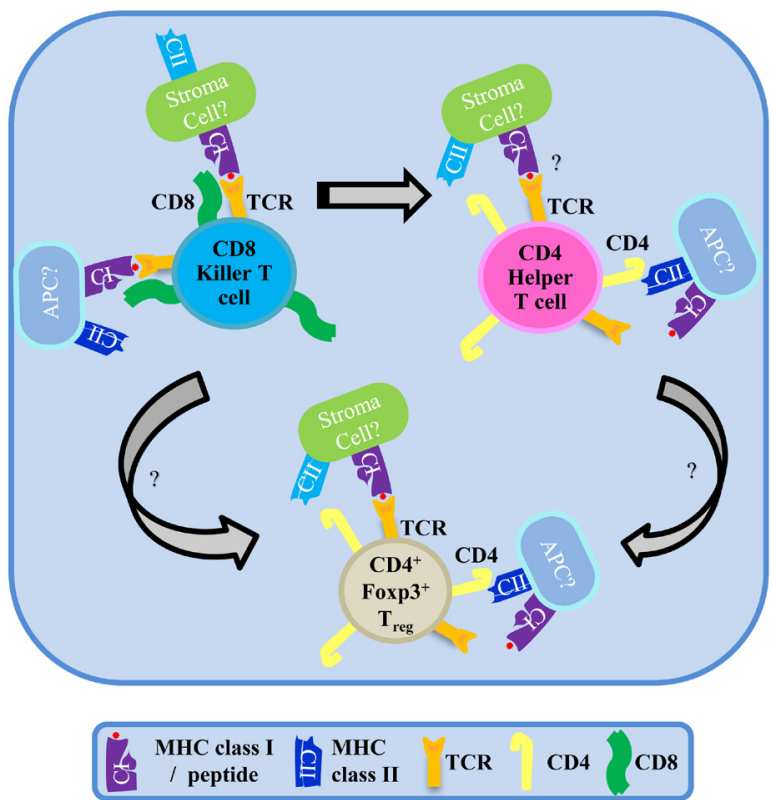

Figure 1: The mismatch to the rescue - a tale of the convert. The CD8 killer T cell lineage converts to MHCIrestricted CD4 Thelper cells and $\mathrm{CD}^{+}{ }^{+} \mathrm{Foxp}^{+}{ }^{+}$suppressor cells in the gut-associated environment. Despite the mismatch of the CD4 co-receptor on the converted CD4 T cells to MHCIrestricted TCR binding, the host-intrinsic plasticity of the CD8 $\mathrm{T}$ cell lineage may serve as an alternative pathway to induce "selfless" tolerance and restore immune balance, especially at the interface with microbiota. 
such a microenvironment triggers $\mathrm{CD} 8$-to-CD4 lineage conversion and generation of MHCI-T $\mathrm{T}_{\text {reg }}$ cells.

In autoimmune diseases, $\mathrm{CD} 4 \mathrm{~T}$ cell depletion has been tested as a potential therapy. In our study, depletion of CD4 T cells in normal mice led to an increased population of CD4 T cells converted from the CD8 lineage. The converted $\mathrm{CD} 4^{+} \mathrm{Foxp} 3^{-}$cells may help activation of CD8 $\mathrm{T}$ cells. Moreover, $\mathrm{MHCI}-\mathrm{T}_{\mathrm{reg}}$ cells might provide a potent antidote against autoimmune damage by CD8 T cells [2]. The conventional MHCII-restricted $\mathrm{CD}^{+} \mathrm{Foxp}^{+}$ $\mathrm{T}$ cells provide dominant tolerance through a variety of mechanisms [4], including cellular contact-based interaction between $\mathrm{T}_{\mathrm{reg}}$ and pathogenic $\mathrm{T}$ cells [5]. One might envisage that MHCI-based recognition facilitates such a direct cellular contact. Of note, MHCI-restricted CD4 T cell clones exist in the natural repertoire of healthy humans [6]. A number of studies have also showed the possibilities of engineering human CD4 T cells recognizing MHCI-presented antigens by transducing MHCI-restricted TCR into CD4 T cells, including CD4 $\mathrm{T}_{\text {reg }}$ cells [7]. Along this direction, understanding the cellular and molecular mechanisms responsible for CD8to-CD4 lineage conversion naturally at a clonal level will greatly benefit therapeutic translation of MHCI-restricted CD4 T cells, particularly MHCI-T $\mathrm{T}_{\text {reg }}$ cells.

Zhibin Chen: Department of Microbiology and Immunology, University of Miami Miller School of Medicine, Miami, Florida, USA

Correspondence to: Zhibin Chen, email zchen@med.miami. edu

Keywords: T lymphocyte, lineage, plasticity, immune tolerance, selfless

Received: July 23, 2015

Published: August 22, 2015

\section{REFERENCES}

1. Burnet FM. Aust J Sci. 1957; 20: 67-69.

2. Lui JB, et al. Cell Rep. 2015; 10: 574-585.

3. Miska J, et al. Eur J Immunol. 2012; 42: 2584-2596.

4. Josefowicz SZ, et al. Annu Rev Immunol. 2012; 30: 531564.

5. Miska J, et al. J Exp Med. 2014; 211: 441-456.

6. Strassman G, et al. J Immunol. 1984; 133: 1705-1709.

7. Plesa G, et al. Blood. 2012; 119: 3420-3430. 